\title{
Network Analysis of the Tel Aviv Mass Transit Plan
}

\author{
Nir Sharav ${ }^{1} \cdot$ Shlomo Bekhor $^{1} \cdot$ Yoram Shiftan $^{1}$
}

Received: 25 December 2016/Revised: 5 November 2017/Accepted: 8 January 2018/Published online: 25 January 2018

(C) The Author(s) 2018. This article is an open access publication

\begin{abstract}
This paper analyzes the strategic transit network plan for the Tel Aviv metropolitan area, using graph theory and other recently developed transit network measures. The different transit modes included in the strategic plan are emphasized by adding weights to distinguish metro lines from light rail lines (LRT). This approach can help compare the combined metro or LRT alternatives of the new Tel Aviv plan to the metro-only alternatives as well as measure the performance relative to the metro systems in other cities around the world. The analysis of the alternative plans in Tel Aviv showed that when metro and LRT lines were treated as homogeneous modes, in which all were considered as metro, the alternatives resembled medium developed metro systems, such as in Barcelona and Washington DC. In contrast, when the distinguished weights were included, the combined metro/LRT alternatives resembled less developed systems, such as in Lyon and Lisbon, and only the metro-only alterative score remained high. The results also showed that the alternatives have regional coverage, and the alternatives with more LRT lines score lower in coverage. The network structure analysis showed that the metro-oriented networks score higher in both directness and connectivity. When using the
\end{abstract}

Yoram Shiftan

shiftan@technion.ac.il

Nir Sharav

nir70@tx.technion.ac.il

Shlomo Bekhor

sbekhor@technion.ac.il

1 Transportation Research Institute, Technion City, Haifa 32000, Israel

Editor: Chun Zhang. weighted measures, the existing plan (LRT-only) scores low on both directness and connectivity. The analysis of the results emphasizes the need for more metro lines in the Tel Aviv metropolitan area. The results also suggest that the analysis of the complex mass transit networks based on graph theory should consider differences in line technology reflected in the line speed and coverage.

Keywords Mass transit - Network analysis - Tel Aviv . Strategic plan

\section{Introduction and Network Measures}

\subsection{Background and Motivation}

The Israeli government prepared a new transport strategic plan for the Tel Aviv metropolitan area (TAMA) that focuses on public transport as the basis for the future metropolitan mobility. Tel Aviv is the largest metropolitan area in Israel and is home to 3.7 million people, accounting for $43 \%$ of the population and $50 \%$ of the employment in Israel. The plan aims to attract passengers to choose public transport by providing premium, reliable, and attractive service.

Allport [1] and Loo and Cheng [15] showed that metro lines should be considered as a major part of the network as the data of 5.0 million population and US $\$ 11,400$ GDP per capita indicate the potential.

The government defined a set of strategic goals for the plan, including transportation, sustainability, social, and economic goals. Based on [17], the plan aimed to ensure that the overall investment in the transit system is similar to Western European cities with developed public transport system (over US\$10,000 per capita), and achieving 
accessibility goals of 45 min transit ride for $60 \%$ of the population and an average transit network speed of $25 \mathrm{~km} / \mathrm{h}$.

Early plans for the TAMA network included only LRT lines [17]. In accordance with Loo and Cheng [15], additional transit modes should be considered. As a result, a new set of transit network alternatives was defined, which includes both metro lines and LRT lines [17].

This paper is focused on the system performance analysis of the proposed network alternatives, emphasizing the differences between the LRT lines and the metro lines. The analysis is based on indicators from graph theory and recent development made by [5] and metro network characteristics analyzed by [6-8]. The network performance measures are described in the next section.

\subsection{Network Performance Measures}

Complex transport networks and especially urban public transportation networks studies using graph theory have underwent much progress in the last decade with the availability of big data and models. Lin and Ban [14] reviewed recent works in network analysis and case studies that used graph theory and measures. They classified and compared the different approaches and measures in various network types such as road network, public transportation network, maritime networks, and aviation. The basic measures covered in their review include node centrality, node degree that represents the number of edges connected to a node, betweenness centrality representing the centrality of the node among other nodes, the degree of the graph clustering, and some additional measures found in recent research. Network indicators such as connectivity, diameter, and accessibility were used in [16].

Levinson [13] used network indicators such as connectivity, treeness, circuity, accessibility, and entropy to study network structure in 50 large US cities. The study found that connectivity increases with metropolitan area population. Larger areas were also found to have fewer miles of road network per capita (probably because of more density), longer edges, and more traffic per edge. The research suggests that larger cities are more connected but have less capacity per capita and longer commute times. Soh et al. [18] analyzed the Singapore public transportation network structure using traditional typological and dynamical (weighted) analysis. They showed that the dynamical analysis (weighted) reveals a more complex picture of the network than the topological network analysis. They used network measures such as the shortest path, degree, range, and strength of the network, as well as the average clustering and centrality.

Table 1 presents the network measures described in [5], who adopted and re-evaluated them to transit network based on the indicators from graph theory. The nodes are defined as vertices, and the links are defined as edges as in the graph theory. These measures are used in this paper with some additional new features described in the Methodology Section.

Derrible and Kennedy [6-8] describe three characteristics of metro networks: state, form, and structure. First, state measures the complexity of the network, whether it is a simple network or a more developed network. Second, form describes the connection between the transit network and the urban environment; thus dividing the transit networks to local coverage, regional coverage, and regional accessibility. Last, structure examines the network structural connectivity (the number of possible transfers) and directness that measures the degree of direct trips possibilities in the network $\delta$.

The optimal design and structure of public transportation began with models of a single line in the early 1970s and seek for optimal frequency and fleet size. Jara-Díaz et al. [12] extended the single line model with financial constraints using urban transit network that can be served with direct services or with transfers. They showed how the financial constraints can affect the optimal line structure and operation and found that the direct line operation with lower frequencies was the preferred outcome.

In recent years, the optimal transit design evolved to city-level network with different urban structures and network representations [2, 11]. Fielbaum et al. [11] presented a model with an urban structure of one CBD and surrounding subcenters. They compared four possible line structures: direct lines, exclusive lines, hub and spoke, and feeder trunk. They found that there is no overall optimal structure and the results depend on many variables. They also found that the direct lines network structure works well with monocentric cities because of fewer transfers, the hubs and spokes structure works well only if the transfer penalty is low, and that the feeder and trunk structure works better in polycentric cities, where the subcenter becomes more attractive and preferential.

Yang et al. 20] investigated the effectiveness of urban rail planning on development in China's megacities. Wang et al. [19] used graph theory to investigate the robustness of metrics in 33 metro networks under random failures. Using the measures, they found that Tokyo and Rome are the most robust networks because of the many options for transfers which provides shorter transfers and more route choices. Feng et al. [10] combined traffic flows with the network measures. They used a multilayer model to analyze traffic flow patterns in the Beijing subway system and concluded that the model can help to better analyze the combined traffic flow and network operation. Cats et al. [3] proposed reliability indicators for measuring network robustness that capture the entire range of possible capacity reduction in metro networks. They developed a robustness 
Table 1 Network measures in Derrible and Kennedy [5]

\begin{tabular}{|c|c|c|}
\hline Measure & Definition & Notes \\
\hline Coverage & $\sigma=\frac{n s \cdot \pi \cdot 0.5^{2}}{A}$ & $\begin{array}{l}\text { The coverage area accessible by the metro network, measured as } 500 \mathrm{~m} \text { from each station divided by } \\
\text { the area serviced (A) }\end{array}$ \\
\hline Directness & $\begin{array}{c}\tau=\frac{k \cdot n L}{\delta} \text { or } \\
\tau=\frac{n L}{\delta}\end{array}$ & $\begin{array}{l}\text { The directness can be described as the total route length }\left(\mathrm{L}=\mathrm{k} \bullet \mathrm{n}_{\mathrm{L}} \text {, where } \mathrm{k} \text { is the average line }\right. \\
\text { length) divided by the maximum number of transfers (representing the longest route possible). A } \\
\text { simpler version considers only the number of lines divided by } \delta \text {. }\end{array}$ \\
\hline $\begin{array}{l}\text { Connectivity (structural } \\
\text { connectivity) }\end{array}$ & $\rho=\frac{v_{c}^{t}-e^{m}}{v^{t}}$ & $\begin{array}{l}\text { The degree of which the network is connected or allowing connections and transfers. It represents the } \\
\text { number of possibilities to transfer. Where } \mathrm{v}_{\mathrm{c}}^{\mathrm{t}}=\text { sum of lines going through a transfer station } \\
\text { minus } 1 \\
\text { To avoid redundant transfers, } \mathrm{e}^{\mathrm{m}} \text { is subtracted. Then, dividing by the total number of transfers, } \mathrm{v}^{\mathrm{t}} \text {, } \\
\text { gives the connectivity index }\end{array}$ \\
\hline Complexity & $\beta=\frac{e}{v}$ & The ratio of edges to vertices \\
\hline Degree of connectivity & $\gamma=\frac{e}{e_{\max }}$ & $\begin{array}{l}\text { Describes how much the network is connected relative to how much it could be connected. It } \\
\text { calculates the number of edges to the potential number of edges (if the network was } 100 \% \\
\text { connected) }\end{array}$ \\
\hline
\end{tabular}

Source: [5]

Where $\mathrm{n}_{\mathrm{L}}=$ number of lines, $\mathrm{n}_{\mathrm{S}}=$ number of stations, $\mathrm{v}=$ number of vertices (nodes) $v=v^{t}+v^{e}, \mathrm{v}^{\mathrm{t}}=$ number of transfer vertices, $\mathrm{v}^{\mathrm{e}}=$ number of end vertices, $\mathrm{e}=$ number of edges (links) $e=e^{s}+e^{m}, \mathrm{e}^{\mathrm{s}}=$ single-use edge (only one line uses this edge), $\mathrm{e}^{\mathrm{m}}=$ multiple-use edge (edges used by two or more lines), $\delta=$ maximum number of transfers needed to go from any vertex to another vertex (can be described as the network diameter). Note that we only consider terminal nodes and transfer nodes connecting at least two lines

assessment model applied to the urban rail network of Amsterdam and concluded that the model and the robustness indicators contribute to a more complete assessment of network robustness.

\section{Methodology}

As indicated in the introduction section, the methodology of this paper is based on recent transit network measures and network characteristics developed by [5-8]. Network measures based on graph theory are a powerful tool to analyze and compare networks in different cities. Cities differ by size, population density, and many other characteristics that affect the transportation network. Each city is unique and different from one another: Some cities have a strong central area, while others have a more dispersed land use. With respect to the transportation system, some cities have an extensive transit network, while others rely on the road network to provide accessibility.

Derrible and Kennedy [5] used the network measures described in Table 1 to analyze and compare metro networks in different cities. They showed that although the cities are very different, these network measures can help categorize cities by the development of their metro network, their complexity, and connectivity.

In this paper, we extend this approach by adding different weights to these network measures to distinguish metro lines from LRT lines. This approach improves the analysis of the proposed alternatives for the Tel Aviv mass transit system. It is especially helpful to compare the combined metro/LRT alternatives to the metro-only alternatives, and compare their expected performance relative to the mass transit systems in other cities around the world. Metro lines have exclusive right of ways in a typical closed network and thus the comparisons are made easier. However, LRT lines have only partial right of way and may operate together with other modes, which make them different and hard to compare to metro networks. These unique characteristics of LRT lines need to be considered when comparing the proposed alternatives of the Tel Aviv plan.

Because we are mainly interested in comparing different measures such as accessibility, coverage, and integration, we decided to differentiate the LRT lines from metro lines by assigning weights to the LRT lines as described in Table 2. The edge factor represents the ratio of LRT speed to metro speed, and thus it represents the difference in accessibility. The factor was calculated based on the average planned line speed. The transfer factor represents the difference in the ease of transfer and thus is the integration of the lines. The station factor represents the difference in coverage or the walk catchment area of the station. El-Geneidy et al. [9] examined the common walk coverage of $400 \mathrm{~m}$ for bus and $800 \mathrm{~m}$ for rail in Montreal, Canada. They showed that actual walk catchment area depends on the mode and level of service (such as wait time) and that the 85 percentile was more than $500 \mathrm{~m}$ for bus and $1200 \mathrm{~m}$ for rail. Daniels and Mulley [4] showed that in Sydney, Australia, the walk distance to station mostly depends on the mode and that people walk further for a faster mode. Similarly, the station walk coverage 
Table 2 LRT lines factors

\begin{tabular}{lll}
\hline LRT lines factor & $\begin{array}{l}\text { Factor } \\
\text { (weights) }\end{array}$ & Notes \\
\hline Edge & 0.65 & Average LRT line speed of $22-23 \mathrm{~km} / \mathrm{h}$ relative to metro average speed of $35 \mathrm{~km} / \mathrm{h}$ \\
Transfer vertex & 1.2 & Transfer penalty of $20 \%$ for LRT transfers (metro to metro $=1.0)$ \\
Line/station & 0.8 & LRT lines and stations are considered $20 \%$ lower in network hierarchy (for metro lines and stations $=1.0$ ) \\
\hline
\end{tabular}

factors in our model are based on common values used for planning (600 m for LRT and 700-800 $\mathrm{m}$ for the faster metro service).

Similar work was carried out by [18], who studied the Singapore rail and bus networks using both typological and dynamical (weighted) analyses. They found that using dynamical analysis (weighted) contributes to the network analysis.

The main phases of the methodology can be described as follows:

- For each of the alternatives, we gathered data required to calculate the network measures (number of lines, stations, vertices, edges, transfer stations...)

- We then calculated the network measures (described in Table 1) for each of the alternatives in accordance with the original measures in [5]. One can look at this calculation as if all the lines are considered as metro lines.

- We repeated the calculation of the network measures, this time using a set of weights for the LRT lines, distinguishing them from the metro lines. The weights intend to represent the main differences between LRT and metro lines in terms of accessibility (LRT edges were considered with lower speed than the metro edges), transfers (metro to metro transfers were considered easier), and finally LRT lines and stations were considered lower in the network hierarchy (see Table 2: LRT lines factors).

- Finally, we compared the measures and network characteristics' plots of state, structure, and form of the plan alternatives relative to other mass transit systems in the world using the network measures with no weights and with weights and summarized the conclusions.

\section{Tel Aviv Mass Transit Plans}

The Israeli government prepared a new strategic plan for the Tel Aviv mass transit network, revising the current plan from 2007. The current plan (alternative A4) included five LRT lines and two BRT lines. Three LRT lines were planned to provide radial service to the center of Tel Aviv.
The other LRT and BRT lines were planned as regional lines. (See appendix for detailed maps).

The plan revision showed that the current plan, A4, is not achieving the strategic goals that the government had set and has significant gaps in each of the performance indicators. The conclusion of the analysis was that the A4 plan is not sufficient and will not provide the necessary level of service required to shift travelers from private cars to public transportation and thus does not support sustainability and growth.

The strategic plan final alternatives for the mass transit system included a new conceptual attentive based entirely on the metro technology providing the best balance between coverage, frequency, speed, and capacity required to serve the demand in the urban area. In addition, new combined alternatives were designed incorporating parts from the current A4 plan with the new network concept. These combined alternatives acknowledge the value of keeping existing plans in order to start constructions within the next two years. The combined plans were also designed to meet the demand and the strategic goals and have a good balance of LOS and costs.

Here is a short description of the final alternatives of the plan. For more details and maps, see the appendix and also [17].

Alternative M5 is a new conceptual one based on five metro lines and the one LRT (red) line that is currently under construction. This plan includes four radial metro lines (additional to the red line) and a ring metro line connecting all the radial lines in a radial web network. All radial lines start at the edge of the urban area and pass through Tel Aviv center to the other edge of the urban area. This alternative is the most developed metro network that also comes with the highest cost of US $\$ 47$ Billion.

Alternative $\mathrm{C} 2$ is a combined network that adds the two most important metro lines (South-North line and a ring line) to the four LRT lines from the exiting plan. This plan keeps the current plan structure and the LRT lines as the backbone of the system. It provides good local service around the center but does not provide good and equal accessibility to all areas in the second ring. $\mathrm{C} 2$ is a combined plan with more LRT lines with a cost estimate of US\$28 Billion. 
Table 3 Tel Aviv plan alternatives-line data

\begin{tabular}{llllrlr}
\hline Plan alternatives & Metro lines & $\mathrm{km}$ & LRT lines & $\mathrm{km}$ & BRT lines & $\mathrm{km}$ \\
\hline C2 & 2 & 71 & 5 & 136 & 4 & 101 \\
C3 & 3 & 144 & 2 & 51 & 5 & 114 \\
M5 & 5 & 144 & 1 & 29 & 3 & 80 \\
A4 & - & - & 5 & 144 & 2 & 42 \\
M100 & 3 & 81 & 1 & 29 & 6 & 117 \\
\hline
\end{tabular}

Alternative C3 is a combined plan with three metro lines, where the backbone of the system is based on the new plan of metro lines and adopts only two LRT lines from the existing plan, A4. C3 plan has $3+$ metro lines, where the plus refers to the third line that includes two branches from the South and from the North joined in a common section along center, with a U-shaped metro line serving the eastbound corridor. C3 is a combined plan with more metro lines and costs US $\$ 39$ Billion.

Alternative M100 is new plan based on the new metro concept that was planned to meet the investment goal of up to NIS100 Billion (US\$26 Billion). It consists of three metro lines: a circular line similar to M5, a radial NorthSouth line, and an Eastbound U-shaped metro line similar to C3 but a little shorter. M100 represents a rather small metro network.

Additional BRT lines were included in all the alternatives. These lines are not included in this analysis.

Table 3 summarizes the different transit lines of each alternative.

\section{Results of the Network Analysis}

Table 4 presents the network data of the plan alternatives described above. Alternative $\mathrm{C} 2$ has the highest number of lines and stations but only two metro lines out of the seven lines. M5 has the highest number of metro lines and the highest average line length. The existing plan, A4, has more stations than $\mathrm{C} 3$ and M5 but all the lines are LRT lines. Finally, M100 has the least number of vertices, edges, and transfer stations.
Table 5 presents the original network measures (without weights) and our version of the modified measures for LRT lines (with weights). The analysis showed that when we used the original measures, $\mathrm{C} 2$ had the highest coverage, complexity, and degree of connectivity; however, when we used the weighted measures, it scored lower than M5 in complexity and connectivity. The M5 network has five metro lines and only one LRT line, and thus it scored highest on almost all measures when we used the weighted measures. The alternative A4 has only LRT lines. It scored the lowest on all weighted measures, except for coverage, where M100 showed lower performance.

Derrible and Kennedy [6-8] presented metro systems development phases ("State") on a graph showing connectivity and complexity. They identified three phases of the metro system development as shown in Fig. 1. The least-developed metro systems are categorized as phase 1, including systems that score up to $\gamma=0.5$ and $\beta=1.3$. Phase 2 (score up to $\gamma=0.6$ and $\beta=1.6$ ) includes metro systems in cities such as Barcelona, Washington DC, Berlin, and Shanghai, while more developed metro systems are categorized as phase 3 (up to $\gamma=0.7$ and $\beta=1.96$ ), including the most developed systems such as Madrid, New York, Paris, Tokyo, and London.

The plot of the alternative plans in Tel Aviv on the graph of system development shows an interesting result. When metro and LRT lines are treated the same (no weights are applied to the LRT lines), all alternatives rank in the phase 2 area. Moreover, alternative $\mathrm{C} 2$ ranks the highest, even higher than Barcelona, Washington DC, and Moscow, and alternative M5 scores second with similar measures to Osaka and Barcelona. Even the exiting plan

Table 4 Tel Aviv plan alternatives-network data

\begin{tabular}{lllllll}
\hline Plan alternatives & Vertices $\mathrm{v}$ & Edges $\mathrm{e}$ & Lines $\mathrm{n}_{\mathrm{L}}$ & Avg. line length $\mathrm{k}$ & ${\text { Stations } \mathrm{n}_{\mathrm{s}}}_{\text {Transfer stations } \mathrm{v}_{\mathrm{t}}}$ \\
\hline C2 & 42 & 69 & 7 & 30 & 249 & 193 \\
C3 & 34 & 46 & 5 & 35 & 195 & 22 \\
M5 & 34 & 51 & 6 & 34 & 214 & 23 \\
A4 & 31 & 42 & 5 & 29 & 113 & 20 \\
M100 & 19 & 25 & 4 & 28 & 11 \\
\hline
\end{tabular}


Table 5 Network measures of the proposed Tel Aviv mass transit alternatives-original measures (no weights) and measures modified to represent LRT lines (with weights)

\begin{tabular}{llllll}
\hline $\begin{array}{l}\text { No weights } \\
\text { Plan alternatives }\end{array}$ & $\begin{array}{l}\sigma=\frac{n s \cdot \pi \cdot 0.5^{2}}{A} \\
\text { Coverage } \sigma\end{array}$ & $\begin{array}{l}\tau=\frac{n L}{\delta} \\
\text { Directness } \tau\end{array}$ & $\begin{array}{l}\rho=\frac{v_{c}^{t}-e^{m}}{v^{t}} \\
\text { Connectivity } \rho\end{array}$ & $\begin{array}{l}\beta=\frac{e}{v} \\
\text { Complexity } \beta\end{array}$ & $\begin{array}{l}\gamma=\frac{e}{e_{\max }} \\
\text { Degree of connectivity } \Upsilon\end{array}$ \\
\hline C2 & 0.13 & 3.5 & 1.00 & 1.6 & 0.58 \\
C3 & 0.10 & 5 & 1.00 & 1.4 & 0.48 \\
M5 & 0.10 & 6 & 1.04 & 1.5 & 0.53 \\
A4 & 0.11 & 2.5 & 0.95 & 1.4 & 0.48 \\
M100 & 0.06 & 4 & 1.09 & 1.3 & 0.49 \\
\hline
\end{tabular}

With weights

\begin{tabular}{lllllr}
\hline Plan alternatives & Coverage $\sigma$ & Directness $\tau$ & Connectivity $\rho$ & Complexity $\beta$ & Degree of connectivity $\Upsilon$ \\
\hline C2 & 0.11 & 3.00 & 0.84 & 1.09 & 0.38 \\
C3 & 0.09 & 4.60 & 0.89 & 1.0 & 90.38 \\
M5 & 0.10 & 5.80 & 0.98 & 1.34 & 0.47 \\
A4 & 0.09 & 2.00 & 0.79 & 0.78 & 0.28 \\
M100 & 0.05 & 3.80 & 0.98 & 1.10 & 0.41 \\
\hline
\end{tabular}

A4 appears in the same area of phase 2 and has similar score to $\mathrm{C} 3$ and M100.

However, when weights were assigned to the LRT lines, the results showed a completely different picture. Only M5 appears in phase 2, its score is lower than Berlin and Barcelona, and it is actually at the lower bound of phase 2 . All the other alternatives appear in phase 1. The exiting plan, A4, scores at the lower bound of phase 1 area (actually even below that). This result is more consistent with the planning team analysis showing significant advantages to the more metro-oriented networks such as M5 and C3 in terms of accessibility and speed.

Figure 2 presents the "Form" of the networks. It shows the average line length and number of stations. The graph is divided into three areas. The "Local coverage" area includes systems with shorter lines and many stations giving more local service. The "Regional accessibility" zone includes systems with longer lines and lower number of stations providing faster services to more-distant suburbs. The third area "Regional coverage" is a mix of the two other types. The alternatives analysis shows that all the plan alternatives are in the area of the "Regional coverage" except for M100 which is in the "Regional accessibility" area (it has shorter lines and less stations). The weighted measures show similar results while the alternatives with more LRT lines score lower in coverage (number of stations).

Figure 3 shows the connectivity and directness of the metro systems representing integration or "Structure." Networks can be exceptional in both directness and connectivity. Paris, Madrid, and Tokyo score high in both connectivity and directness and are labeled as integrated networks. London and Washington score high in directness but little lower in connectivity. The TAMA plan alternatives analysis shows that the metro-oriented networks (M5, C3, and M100) score higher in both directness and connectivity. When using the weighted measures, A4 scores low in both directness and connectivity with $\mathrm{C} 2$ scoring lower on connectivity.

\section{Summary and Conclusions}

This paper analyzes the new strategic plan for the Tel Aviv mass transit network. The methodology used in this paper is based on the recent transit network measures and network characteristics developed by [5-8]. This paper added unique features to distinguish metro lines from light rail lines (LRT). This approach can help compare the combined metro/LRT alternatives to metro-only networks and measure the performance relative to mass transit systems in other cities in the world.

As described in [6-8], three system development phases were identified. The analysis of the alternative plans in Tel Aviv on the graph of system development showed an interesting result. When metro and LRT lines are treated similarly (no weights are applied to the LRT lines), all alternatives appeared in "Phase 2." Moreover, alternative C2 ranked the highest, even higher than Barcelona, Washington DC, and Moscow. The metro-only alternative (M5) scored second with similar measures to Osaka and Barcelona. Even the exiting plan, A4, appeared in "Phase 2" and has a similar score to C3 and M100. In contrast, when weights were assigned to the LRT lines, the results showed a completely different picture. Only the pure metro alternative (M5) appeared in "Phase 2." All the other 
Fig. 1 Network development (state). Note The TA network alternatives are presented on top of original graph from [6-8]
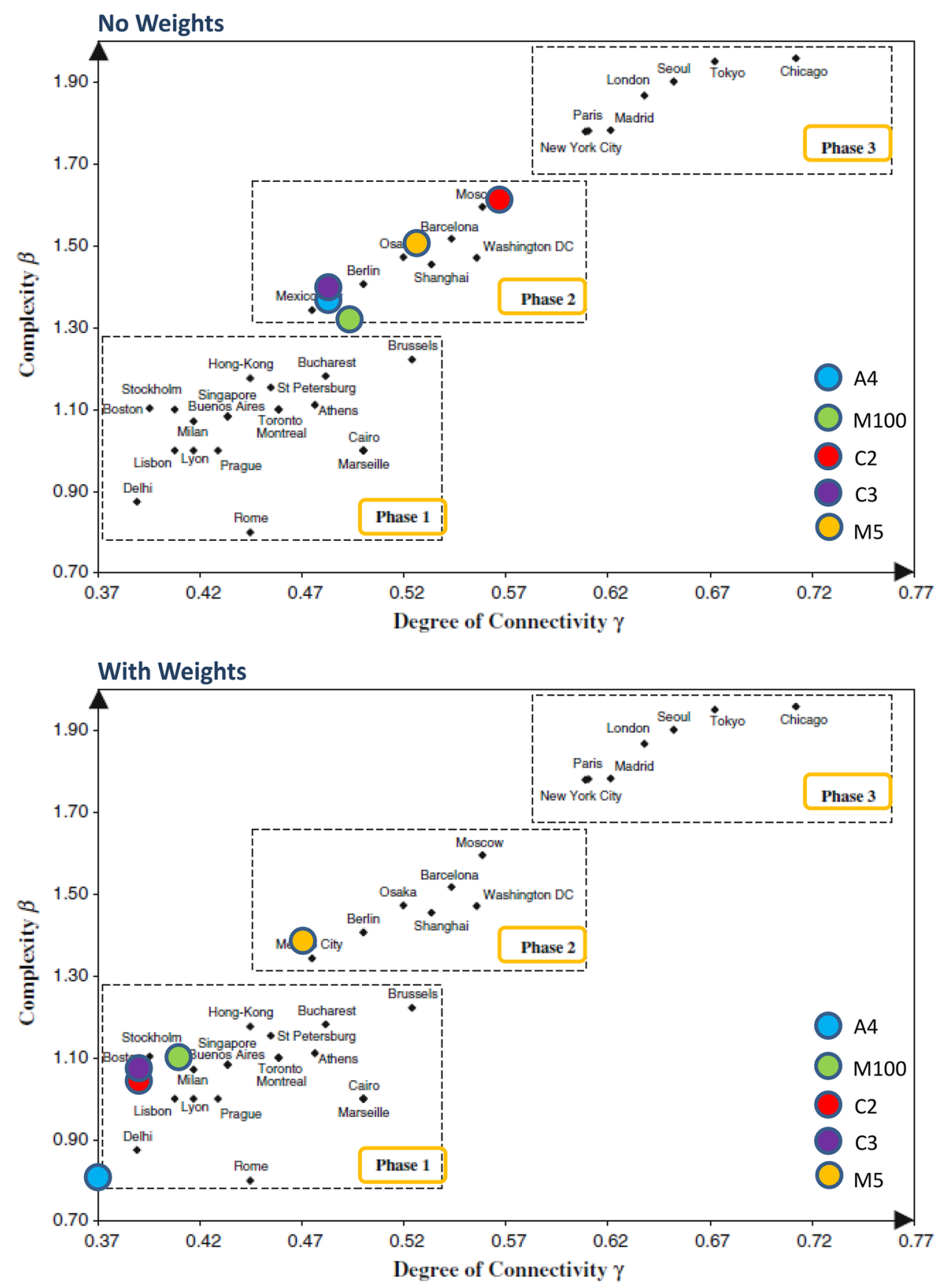

alternatives appeared in "Phase 1," similar to Lyon, Lisbon, and Athens. Moreover, the exiting plan (LRT only), A4, appeared below the lower bound of "Phase 1," suggesting that it might be inferior to the new proposed alternatives including metro lines.

The results show that all the network alternatives have regional coverage except for M100, because this network alternative has shorter lines and hence less coverage. Using the proposed weighted measures, we obtained similar results, but the alternatives with more LRT lines scored lower in coverage. This is explained by the differentiated passenger behavior: according to the literature, passengers are willing to walk longer distances to a faster mode [4, 9].

The analysis of the network structure shows that the metro-oriented networks (M5, C3, and M100) score higher in both directness and connectivity, meaning that they are more integrated. When using the weighted measures, A4 scores low in both directness and in connectivity with $\mathrm{C} 2$ scoring lower on connectivity.

The results also suggest that the analysis of complex mass transit networks based on graph theory should consider differences in line technology. This result is similar to 
Fig. 2 Network form
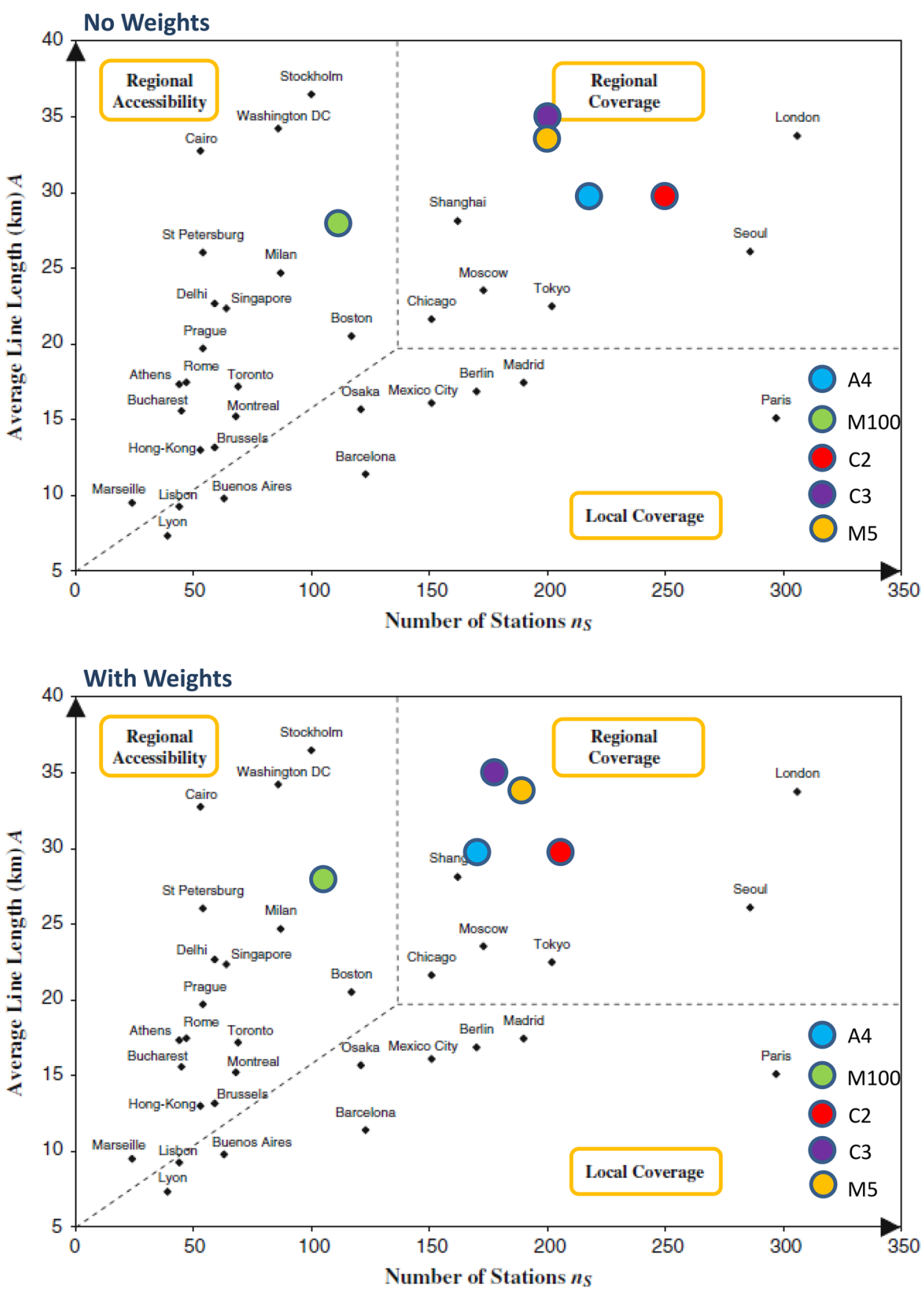

the findings of [18] in the Singapore network showing different results when using weighted measures and analyzing public transportation systems. In this paper, we used line speed to represent the difference in accessibility between metro and LRT. We used transfer factor to represent the difference in integration, and station factor to represent the difference in coverage. However, future research should explore better considerations and methods to develop these weights as well as run sensitivity analysis regarding these weights in various cities.
As indicated in the introduction and methodology section, the contribution of the paper is to include weights in order to account for different transit modes (LRT and metro). In this aspect, the LRT weights proposed in the paper are specific to the Tel Aviv network. Further research should be performed to propose a general calculation for the LRT (or other modes) weights.

We also suggest that future research will include other transit modes (such as BRT) and more features (such as line capacity, frequency, and others) distinguishing the difference in line technology. 
Fig. 3 Network integration (structure)
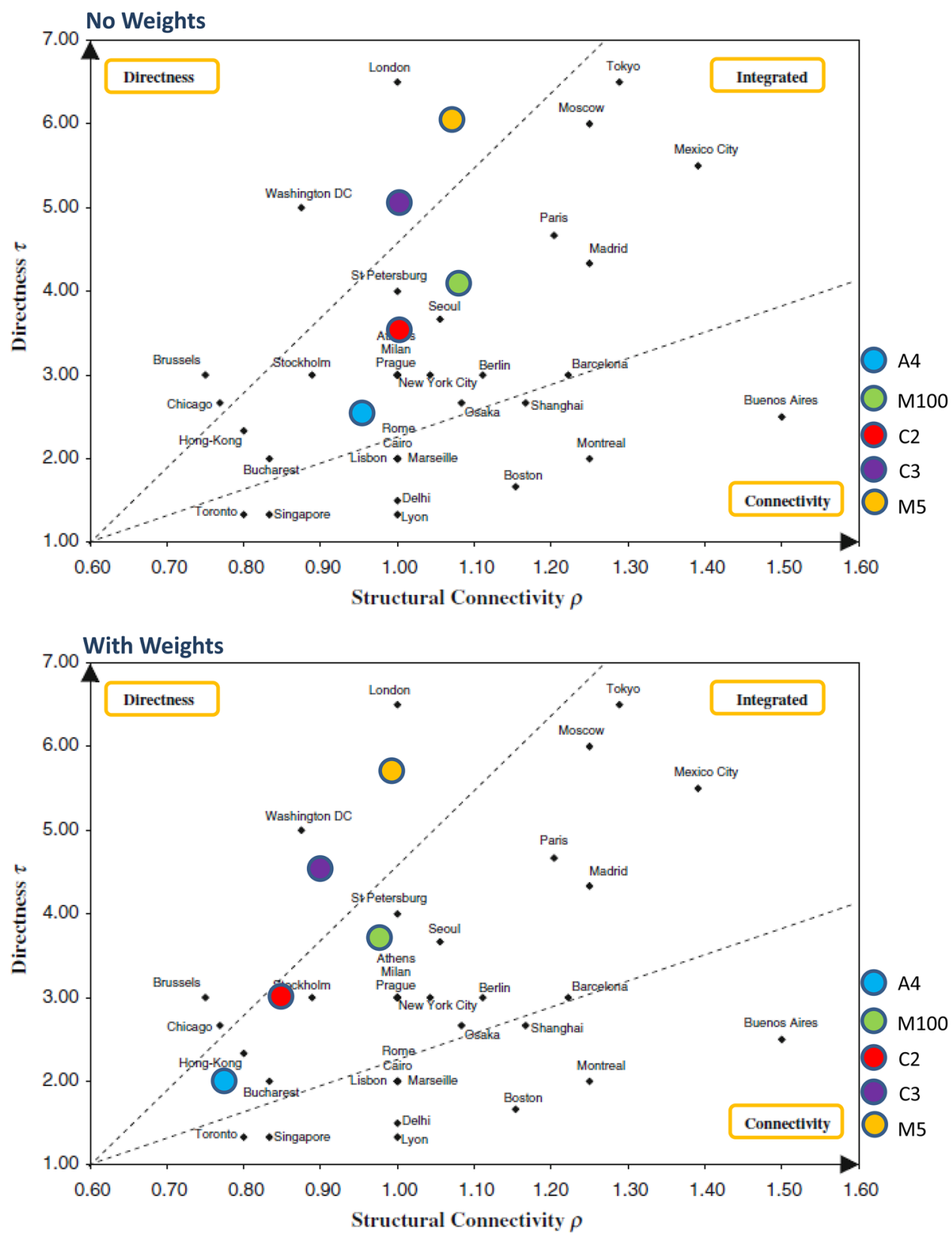

Acknowledgements The authors would like to thank Yitzhak Gelber, Yulia Rubinstein, and Marcos Szeinuk for their help preparing this paper.

Open Access This article is distributed under the terms of the Creative Commons Attribution 4.0 International License (http:// creativecommons.org/licenses/by/4.0/), which permits unrestricted use, distribution, and reproduction in any medium, provided you give appropriate credit to the original author(s) and the source, provide a link to the Creative Commons license, and indicate if changes were made. 


\section{Appendix}
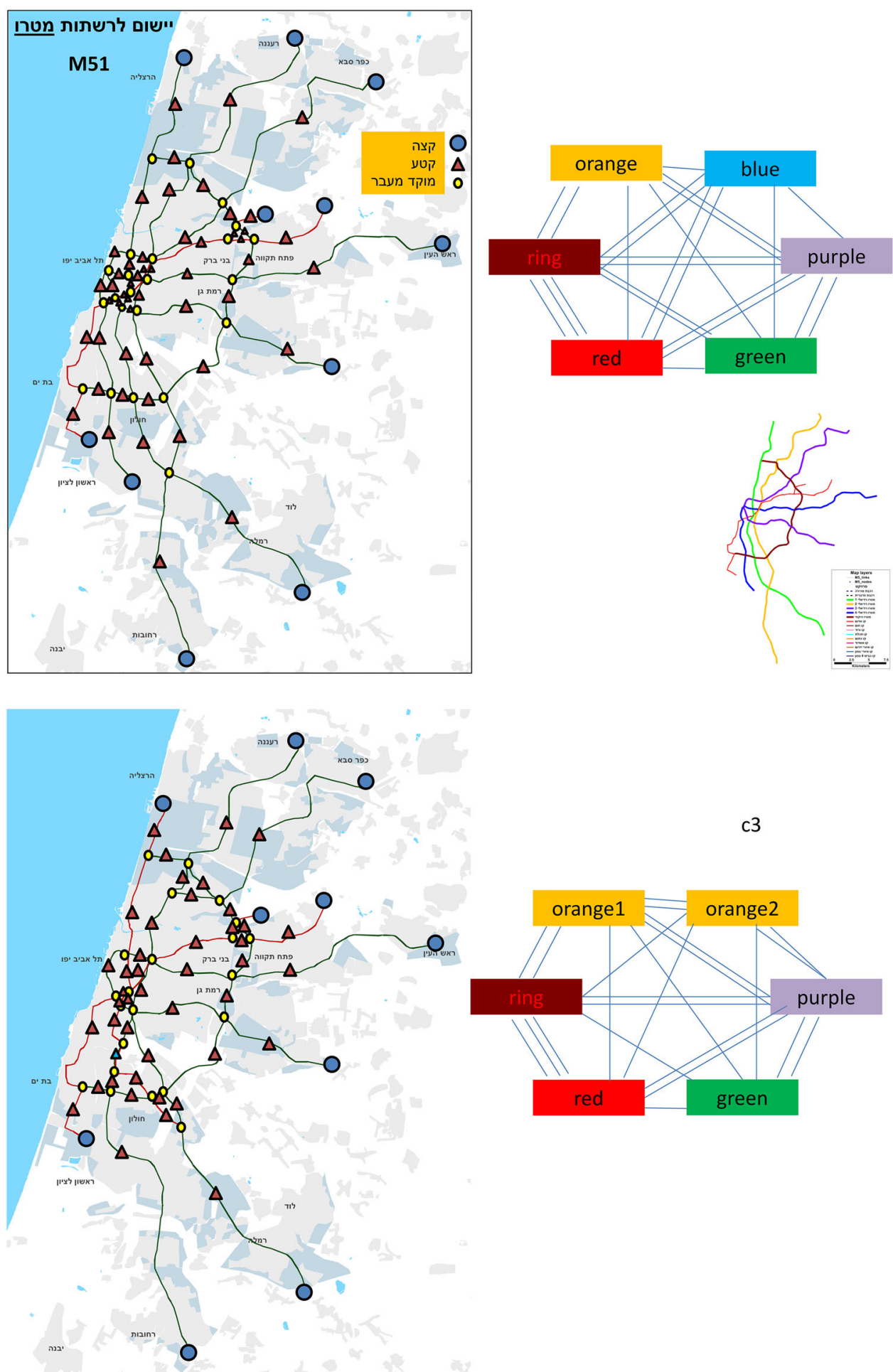

c3
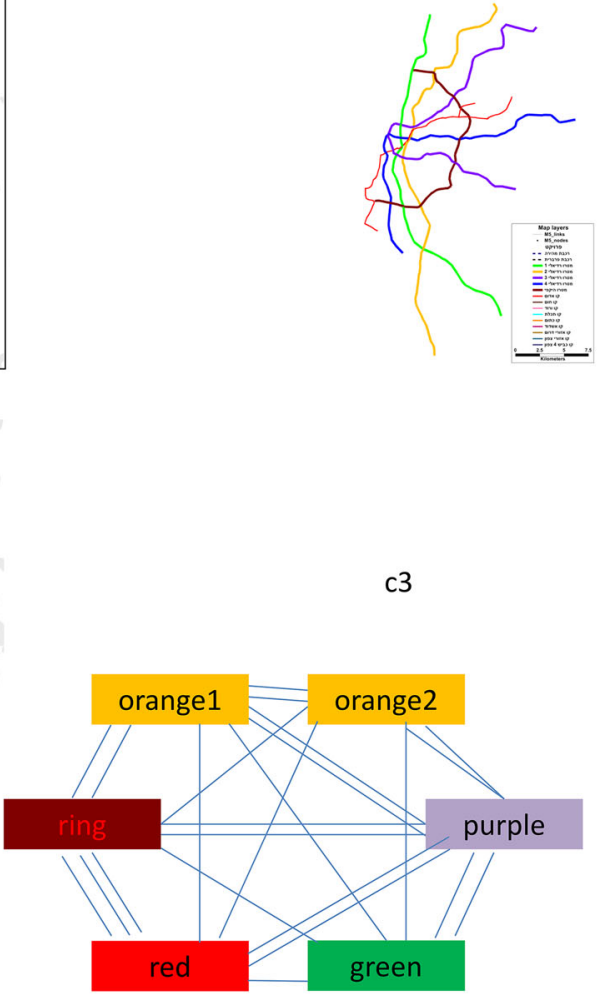

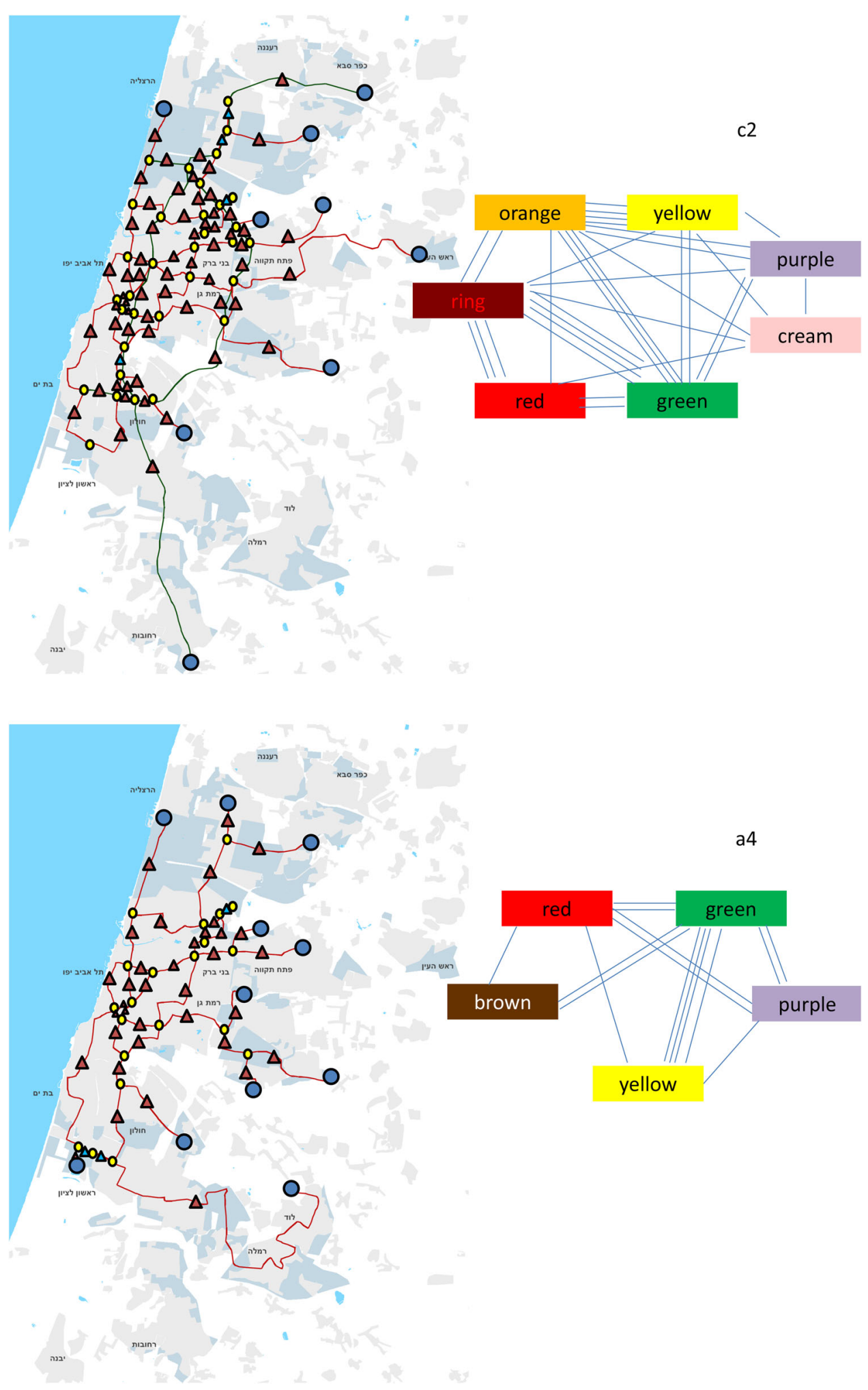


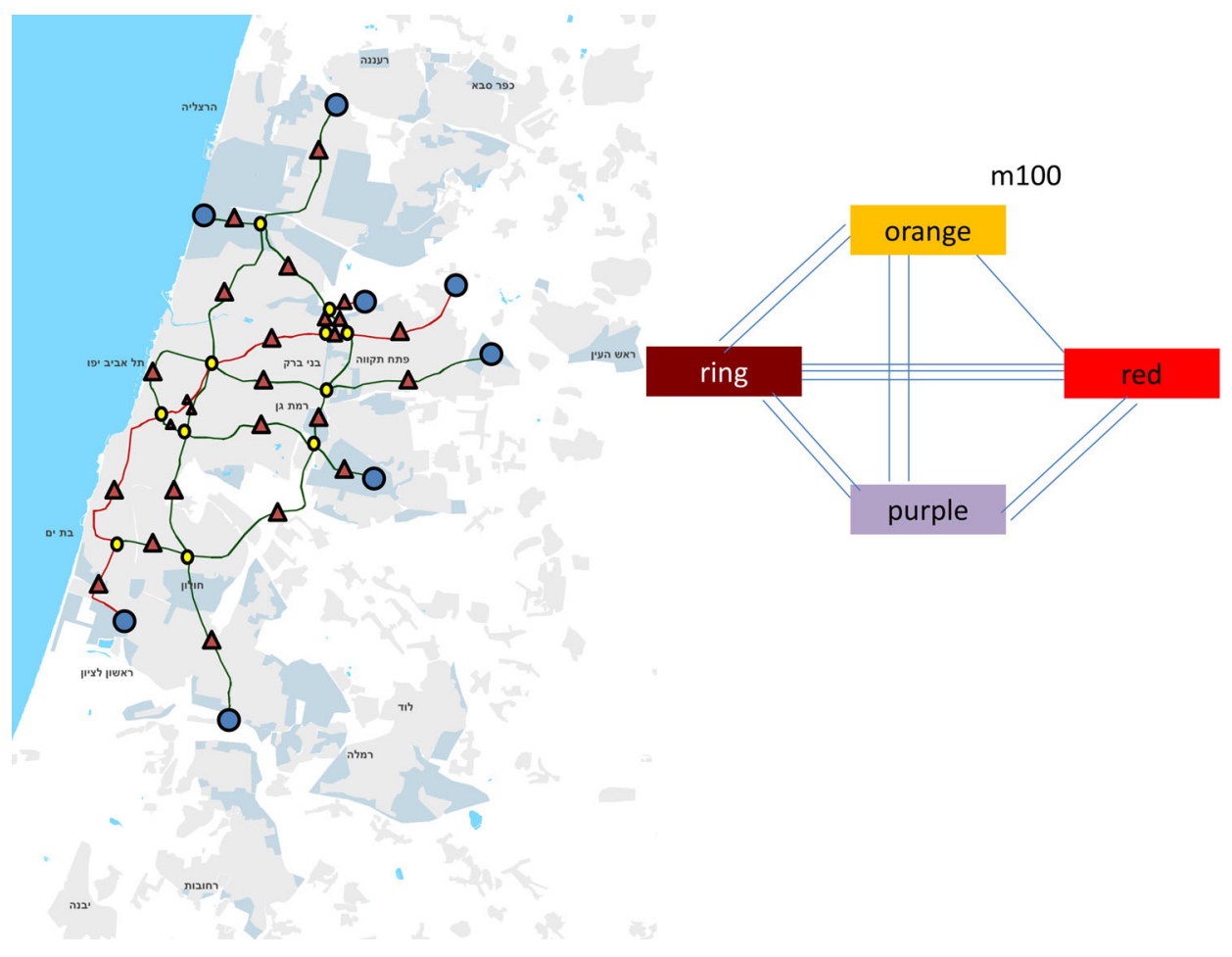

\section{References}

1. Allport R (1990) The metro: determining its viability. In: Margaret $\mathrm{JH}$ (ed) Developing world transport. Grosvenor Press International, London, pp 64-69

2. Badia H, Estrada M, Robusté F (2016) Bus network structure and mobility pattern: a monocentric analytical approach on a grid street layout. Transp Res B Methodol 93:37-56. https://doi.org/ 10.1016/j.trb.2016.07.004

3. Cats O, Koppenol GJ, Warnier M (2017) Robustness assessment of link capacity reduction for complex networks: application for public transport systems. Reliab Eng Syst Saf 167(June):544-553. https://doi.org/10.1016/j.ress.2017.07.009

4. Daniels R, Mulley C (2011) Explaining walking distance to public transport: the dominance of public transport supply. In: World symposium on transport and land use research. The University of Sydney, Whistler, pp 1-22

5. Derrible S, Kennedy C (2009) network analysis of world subway systems using updated graph theory. Transportation Research Record: Journal of the Transportation Research Board, No. 2112, Transportation Research Board of the Natioanl Academies, Washington DC, pp 17-25

6. Derrible S, Kennedy C (2010) Characterizing metro networks: state, form, and structure. Transportation 37:275-297

7. Derrible S, Kennedy C (2010b) Evaluating, comparing, and improving metro networks. Transportation Research Record: Journal of Transportation Research Board, No. 2146, Transportation Research Board of the National Academies. Washington DC, pp 43-51

8. Derrible S, Kennedy C (2010) The complexity and robustness of metro networks. Phys A 389:3678-3691

9. El-Geneidy A, Grimsrud M, Wasfi R (2014) New evidence on walking distances to transit stops: identifying redundancies and gaps using variable service areas. Transportation 41(1):193-210
10. Feng J, Li X, Mao B, Xu Q, Bai Y (2017) Weighted complex network analysis of the Beijing subway system: train and passenger flows. Phys A 474:213-223. https://doi.org/10.1016/j. physa.2017.01.085

11. Fielbaum A, Jara-Diaz S, Gschwender A (2016) Optimal public transport networks for a general urban structure. Transp Res B Methodol 94:298-313. https://doi.org/10.1016/j.trb.2016.10.003

12. Jara-Díaz SR, Gschwender A, Ortega M (2014) The impact of a financial constraint on the spatial structure of public transport services. Transportation 41(1):21-36. https://doi.org/10.1007/ s11116-013-9461-x

13. Levinson D (2012) Network structure and city size. PLoS ONE 7(1):e29721

14. Lin J, Ban Y (2013) Complex network topology of transportation systems. Trans Rev 33(6):658-685

15. Loo BP, Cheng AH (2010) Are there useful yardsticks of population size and income level for building metro systems? Some worldwide evidence. Cities 27:299-306

16. Rodrigue J-P, Slack B, Comtois C (2013) The geogrphy of transport systems. Routledge, New York

17. Sharav N, Shiftan Y, Szeinuk M (2016) Does your city needs a metro- the case study of Tel Aviv (submition)

18. Soh H, Lim S, Zhang T, Fu X, Lee GK, Hung TG, Wong L (2010) Weighted complex network analysis of travel routes on the Singapure public transportation system. Phys A 389:5582-5863

19. Wang X, Koç Y, Derrible S, Ahmad S, Pino W, Kooij R (2017) Multi-criteria robustness analysis of metro networks. Phys A 474:19-31

20. Yang J, Quan J, Yan B, He C (2016) Urban rail investment and transit-oriented development in Beijing: can it reach a higher potential? Transp Res Part A Policy Pract 89:140-150. https:// doi.org/10.1016/j.tra.2016.05.008 